\title{
Survival Outcomes in Patients with Breast Cancer: Low Volume Single Center Study
}

\author{
Seong Uk Jang, M.D., Yoo Seok Kim, M.D., Kweon Cheon Kim, M.D., Ph.D. \\ Department of Surgery, Chosun University College of Medicine, Gwangju, Korea
}

Purpose: Breast cancer is the second most common malignancy in Korean women, and its incidence has increased rapidly. The purpose of this study was to evaluate the treatment outcomes of patients with breast cancer treated at a low volume center. Methods: We retrospectively analyzed 401 patients with stage I-III breast cancer at Chosun University Hospital from January 1998 to December 2013. We reviewed medical records including clinical information and pathologic reports. Results: We found 401 cases of newly diagnosed breast cancer, including 5 (1.2\%) male breast cancer patients. According to histological features, there were 43 cases (ductal carcinoma in situ (DCIS): 35 cases [8.7\%], lobular carcinoma in situ (LCIS): 8 cases [2.0\%]) of non-invasive carcinoma, 350 cases (87.3\%) of invasive carcinoma, 2 cases $(0.5 \%)$ of Paget's disease, 1 case $(0.2 \%)$ of malignant phyllodes tumor, 3 cases $(0.7 \%)$ of sarcoma, and 2 cases $(0.5 \%)$ of other types. With respect to disease stage, 117 (29.2\%) patients were in stage I, $176(43.9 \%)$ in stage II, and $78(19.4 \%)$ in stage III. The type of surgical approaches were breast-conserving surgery $(153,38.2 \%)$, modified radical mastectomy $(243,60.6 \%)$, wide excision $(4,1.0 \%)$, and others $(1$, $0.2 \%$ ). Five-year disease-free survival rates were 100\% (stage 0), 95.4\% (stage I), $91.4 \%$ (stage Ila), $75 \%$ (stage Ilb), $78 \%$ (stage Illa), $50 \%$ (stage IIlb), and $59.2 \%$ (stage Illc). Overall survival rates were 100\% (stage 0), 96.2\% (stage l), $94.3 \%$ (stage lla), $85.4 \%$ (stage llb), $84.8 \%$ (stage IIla), 50\% (stage IIIb), and $55.5 \%$ (stage IIIc). Conclusion: Although the patients were treated at a low-volume center, their favorable survival outcomes are notable. Further multicenter (low-volume centers) research is warranted.

Key Words: Breast, Hospitals, Neoplasms, Survival

\section{INTRODUCTION}

Breast cancer is one of the most common malignancies among Korean women after thyroid cancer. Korea has a low breast cancer incidence rate (43.8 per 100,000 women-years in 2009) [1], which is increasing at a rapid rate. The crude incidence rate of breast cancer among Korean women in 2015, including carcinoma in situ, was 88.1 per 100,000, a significant increase from 26.3 in 2000 [2]. In Korea, the five-year survival rate is $91.2 \%$ (89.2\% in the US and $89.1 \%$ in Japan), showing an increase from $83.2 \%$ (1996-2000) to 88.5\% (2001-2005) [3]. The incidence and death rates of breast cancer differ slightly among countries. Worldwide, from 1980 to 2010, the number of breast cancer cases has been steadily increasing, and the incidence of breast cancer is increasing at an annual rate of $3.1 \%$, and mortality from

Correspondence: Yoo Seok Kim, M.D.

Department of Surgery, Chosun University College of Medicine, 365 Pilmun daero, Dong-gu, Gwangju 61452, Korea

Tel: + 82-62-220-3249, Fax: +82-62-228-3441, E-mail: quintet64@chosun.ac.kr This study was supported by research funds from Chosun University Hospital, 2016.

Received: Sep 13, 2019 Revised: Oct 20, 2019 Accepted: Feb 22, 2020 breast cancer is increasing at an annual rate of $1.8 \%$. Breast cancer is the leading cause of cancer-related deaths with an estimated 1.7 million cases and 521,900 deaths in 2012. Breast cancer accounts for 25\% of all cancer cases and $15 \%$ of all cancer-related deaths among women [4].

In future, screening that includes mammography and ultrasonography can help reduce the incidence of advanced cancer and breast cancer mortality owing to early detection of breast cancer [5]. Many randomized controlled trials (RCTs) have shown that routine screening mammography for women aged over 40 years can help early detection of breast cancer and decrease breast cancer mortality by over $30 \%$ [6]. The effect of screening in reducing breast cancer mortality persists with long-term follow-up. The effect is age-dependent: the highest effect is seen in women aged 55-69 years and lowest, in women aged 50-54 years, at randomization [7].

Hospital volume can be categorized into three levels : low volume ( $<148$ cases/year), moderate volume (148-298 cases/year), and high volume (> 298 cases/year) [8]. Our center is a low-volume center. The purpose of this study was to analyze breast cancer treatment outcomes in a low-volume center. 


\section{METHODS}

This study was approved by Institutional Review Board at the chosun university hospital (IRB No. 2019-08-024-001). Data were collected from the records of patients who were newly diagnosed with primary breast cancer during the period of January 1998 to December 2013 at Chosun university hospital, South Korea. Clinical and pathological data were extracted from electric medical records. The following parameters were extracted: surgical method, pathological stage (American Joint Committee on Cancer [AJCC], eighth edition), tumor, node, metastasis (TNM) stage, histological categorization, hormone receptor status, and human epidermal growth factor receptor 2 (HER2) positivity. Survival analysis was performed for disease-free survival (DFS) and overall survival (OS) by the Kaplan-Meier method based on anatomic stage. The level of significance was set at $5 \%$ for all statistical tests. All statistical analyses were performed using SPSS21.0 (SPSS Inc., Chicago, USA)

\section{RESULTS}

\section{Number, sex, and age distribution of patients}

In our center, there were 401 cases of newly diagnosed breast cancer (Table 1), with 5 (1.2\%) male breast cancer patients. The median age was 52 years. The largest number of newly diagnosed patients was in the $50-59$ years age group (126 cases, $31.4 \%$ ), followed by the $40-49$ years age group (122 cases, 30.4\%) (Figure 1).

\section{Surgical approach and staging}

The types of surgical approaches adopted at our center from 1998 to 2013 were breast-conserving surgery (BCS) (153, 38.2\%), modified

Table 1. Age distribution

\begin{tabular}{lc}
\hline Age $(\mathrm{yr})$ & No. $(\%)$ \\
\hline $20-29$ & $3(0.7)$ \\
$30-39$ & $289(7.0)$ \\
$40-49$ & $122(30.4)$ \\
$50-59$ & $126(31.4)$ \\
$60-69$ & $75(18.7)$ \\
$70-79$ & $42(10.5)$ \\
$80-89$ & $4(1.0)$ \\
$90-99$ & $1(0.2)$ \\
Total & $401(100.0)$ \\
\hline
\end{tabular}

radical mastectomy (MRM) $(243,60.6 \%)$, wide excision $(4,1.0 \%)$, and others $(1,0.2 \%)$.

In all, 117 patients (29.2\%) were in stage I, $176(43.9 \%)$ in stage II, and 78 (19.4\%) in stage III of the disease. Stage IIa was the most common disease stage, with 120 cases (32.2\%), followed by stage I (29.2\%) (Table 2).

\section{Pathological features and biomarkers}

Among the 401 patients, invasive ductal carcinoma was the most common diagnosis, with 346 cases (86.3\%), followed by ductal carcinoma in situ (DCIS), with 35 cases (8.7\%). According to histological type, there were 43 cases (DCIS: 35, LCIS: 8) of non-invasive carcinoma, 350 cases $(87.3 \%)$ of invasive carcinoma, 2 cases $(0.5 \%)$ of Paget's disease, 1 case $(0.2 \%)$ of malignant phyllodes tumor, 3 cases $(0.7 \%)$ of sarcoma, and 2 cases $(0.5 \%)$ of other types (Table 2 ).

Estrogen-receptor (ER)- and progesterone-receptor (PR)-positive cases accounted for $53.4 \%$ of all cases. Tumors types that were positive for both hormone receptors were observed in 144 cases (35.9\%) and those that were negative for both hormone receptors, in 129 cases $(32.2 \%)$ (Table 2).

\section{Five-year survival and overall survival}

The five-year DFS rates were 100\% (stage 0), 95.4\% (stage I), 91.4\% (stage IIa), 75\% (stage IIb), 78\% (stage IIIa), 50\% (stage IIIb), and 59.2\% (stage IIIc) (Log rank $p<0.001)$ (Figure 2). The OS rates were $100 \%$ (stage 0), 96.2\% (stage I), 94.3\% (stage IIa), 85.4\% (stage IIb), 84.8\%

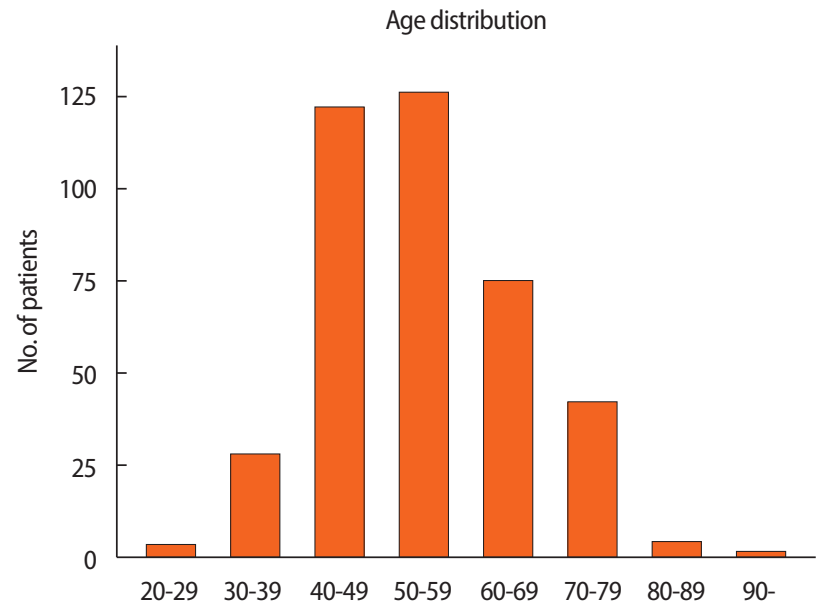

Figure 1. The largest number of newly diagnosed cases was in the 50 to 59 years age group. 
Table 2. Clinicopathologic features in all patients $(n=401)$

\begin{tabular}{lc}
\hline Characteristic & No. (\%) \\
\hline Sex & \\
Female & $396(98.8)$ \\
Male & $5(1.2)$ \\
Type of surgery & \\
Breast conserving surgery & $153(38.2)$ \\
Modified radical mastectomy & $243(60.6)$ \\
Wide excision & $4(1.0)$ \\
Others & $1(0.2)$ \\
Pathologic diagnosis & \\
Ductal carcinoma in situ & $35(8.7)$ \\
Invasive ductal carcinoma & $346(86.3)$ \\
Lobular carcinoma in situ & $8(2.0)$ \\
Invasive lobular carcinoma & $4(1.0)$ \\
Paget's disease & $2(0.5)$ \\
Malignant phyllodes tumor & $1(0.2)$ \\
Sarcoma & $3(0.7)$ \\
Others & $2(0.5)$ \\
Hormone receptor status & \\
ER+/PR+ & $144(35.9)$ \\
ER+/PR- & $38(9.5)$ \\
ER-/PR+ & $32(8.0)$ \\
ER-/PR- & $129(32.2)$ \\
Unknown & $58(14.5)$ \\
Stage & \\
Stage 0 & $30(7.5)$ \\
Stage I & $117(29.2)$ \\
Stage lla & $129(32.2)$ \\
Stage Illb & $47(11.7)$ \\
Stage Illa & $47(11.7)$ \\
Stage Illb & $3(0.7)$ \\
Stage Illc & $28(7.0)$ \\
\hline &
\end{tabular}

$E R=$ estrogen receptor; $\mathrm{PR}=$ progesterone receptor.

(stage IIIa), 50\% (stage IIIb), and 55.5\% (stage IIIc) (Log rank $p<0.001)$ (Figure 3).

\section{DISCUSSION}

The number of newly diagnosed breast cancer patients in South Korea has been increasing [9]. Although the reasons for the increasing trend are not completely understood, it likely reflects birth-cohort effects, changes in reproductive factors (early menarche, late childbearing, and having fewer children), diet (i.e., consumption of high-calorie foods), obesity, and the introduction of screening programs [10]. In Korea, the median age of newly diagnosed patients in 2010 was 49 years, which was slightly higher than that in the past, but is more than 10 years lower than that in the US [11]. The median age reported in

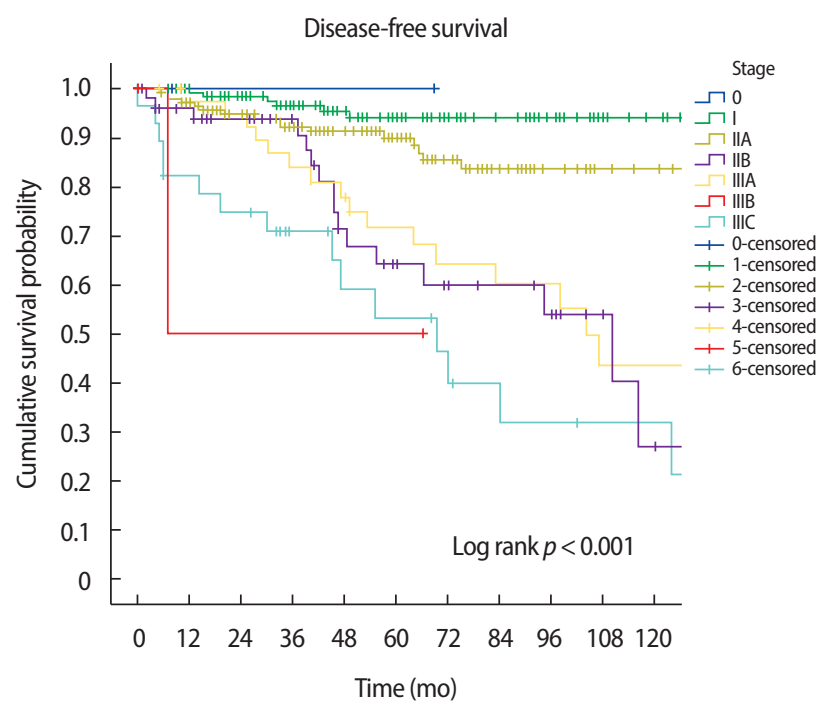

Figure 2. Disease-free survival according to tumor stage.

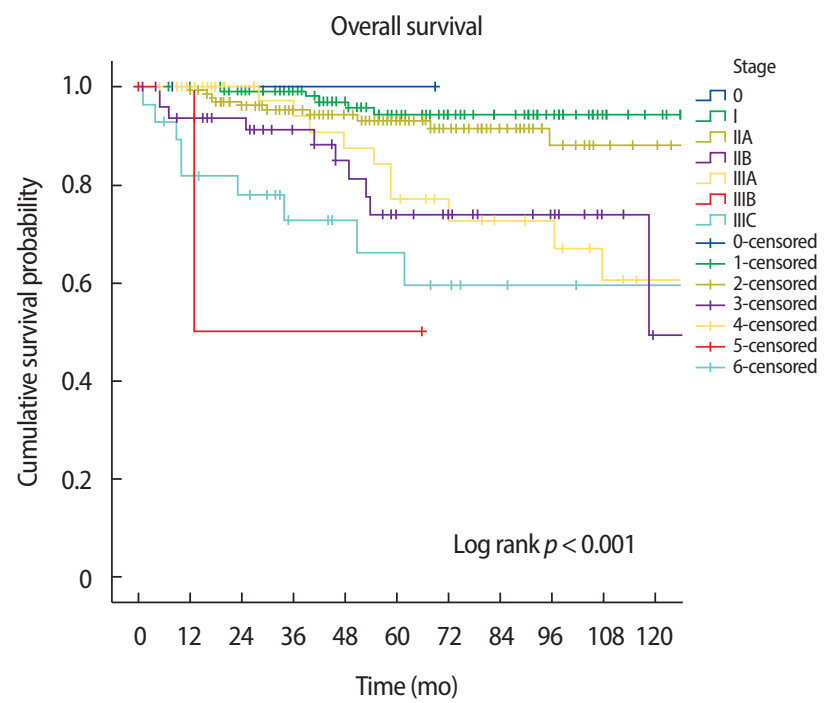

Figure 3. Overall survival according to tumor stage.

our hospital is similar to that in all of Korea.

Breast cancer in men is being diagnosed at early stages. The percentage of breast cancer cases diagnosed at stage 0 or I has consistently increased from $7.0 \%$ to $31.1 \%$ in 2002 and from $14.8 \%$ (111.4\% increase; $\left.\mathrm{R}^{2}=0.847, p<0.001\right)$ to $43.3 \%\left(25.8 \%\right.$ increase; $\left.\mathrm{R}^{2}=0.894, p<0.001\right)$ in 2015 [12]. In breast cancer RCTs, there was a decrease in the risk of being diagnosed with advanced breast cancer because of mammographic screening programs [13]. The ratio of early breast cancer (stages 0 and I) cases increased from $38.1 \%$ of all breast cancer cases in 2002 to $55.7 \%$ in 2014 [14]. At our center, the percentage of cases diag- 
nosed at stage 0 or 1 was $37.6 \%$ (147 patients) from 1998 to 2013 .

In Korea, the ratio of BCS is found to surpass that of mastectomy. The proportion of patients undergoing BCS is twice that of patients undergoing mastectomy patients (67.2\% vs. 32.3\%) [15]. However, in recent years, the patients undergoing mastectomy has been slightly increasing [16]. At our hospital, MRM is still the most common breast cancer surgery. In recent years, the proportion of BCS has shown a steady increased.

In our hospital, there were 214 (43.4\%) ER-positive or PR-positive breast cancer patients (Table 2). There has been a recent increase in the proportion of ER-positive breast cancer cases worldwide [11]. A similar trend has been observed in Korea and Japan [17]. There are some reports on the reasons behind the increase in ER-positive breast cancer [18]. Yip et al. [19] reported that ER positivity was significantly associated with early stage (I, II) and low-grade breast cancers. Ko et al. [11] examined this recent trend, noting that the incidence of ER-positive breast cancers will increase, the incidence of ER-negative breast cancers will decrease, and the overall incidence of breast cancer will remain steady.

Breast cancer is the second most common cause of death and accounts for $14 \%$ all new cancer diagnoses in women [4]. The five-year relative survival rates (\%) for breast cancer in all races increased from 84\% during the mid-1970s to $91 \%$ in 2005-2011 [4].

Especially, patients with ER/PR-positive breast cancer experienced significant survival gains across all stages, and the highest annual increase in survival rates was reported for women is stages III and IV of the disease [20].

In Korea, the five-year survival rate is $91.2 \%$ (89.2\% in the US, $89.1 \%$ in Japan), increasing from $83.2 \%$ (1996-2000) to $88.5 \%$ (2001-2005) [11]. For each stage, the five-year OS rates were 98.3\% (stage 0), 96.6\% (stage I), 91.8\% (stage II), 75.8\% (stage III), and 34.0\% (stage IV) [14].

The five-year DFS rates in our hospital were 100\% (stage 0), 95.4\% (stage I), 91.4\% (stage IIa), 75\% (stage IIb), 78\% (stage IIIa), 50\% (stage IIIb), and 59.2\% (stage IIIc). This result corresponds to that of a previous large-scale clinical study [3]. However, the proportion of stage III cases is slightly different from that reported in the large-scale clinical study due to the small number of patients in our study.

In many studies, high-volume centers were found to be independent predictors of improved OS, and a direct inverse relationship between volume and mortality from high-risk surgical procedures for cancer has been reported [8].

Our study has a few limitations such as the small sample size. However, we have shown favorable survival outcomes despite our center being a low-volume one. Although further investigation involving collaboration between different low-volume centers is needed, patients can be treated at low-volume centers with excellent outcomes.

\section{CONFLICT OF INTEREST}

The authors declare that they have no competing interests.

\section{REFERENCES}

1. Shin HR, Joubert C, Boniol M, Hery C, Ahn SH, Won YJ, et al. Recent trends and patterns in breast cancer incidence among Eastern and Southeastern Asian women. Cancer Causes Control 2010;21: $1777-85$

2. Korean Breast Cancer Society. Breast cancer facts \& figures 2018. Seoul: Korean Breast Cancer Society; 2018.

3. Krieger N, Bassett MT, Gomez SL. Breast and cervical cancer in 187 countries between 1980 and 2010. Lancet 2012;379:1391-2.

4. Torre LA, Bray F, Siegel RL, Ferlay J, Lortet-Tieulent J, Jemal A. Global cancer statistics, 2012. CA Cancer J Clin 2015;65:87-108.

5. Geisel J, Raghu M, Hooley R. The role of ultrasound in breast cancer screening: the case for and against ultrasound. Semin Ultrasound CT 2018;39:25-34.

6. Weedon-Fekjaer H. Effectiveness of population-based service screening with mammography for women ages 40 to 49 years: evaluation of the Swedish mammography screening in young women (SCRY) cohort. Cancer 2012;118:1169.

7. Nystrom L, Andersson I, Bjurstam N, Frisell J, Nordenskjold B, Rutqvist LE. Long-term effects of mammography screening: updated overview of the Swedish randomised trials. Lancet 2002;359: 909-19.

8. Rachel A. Greenup, Samilia Obeng-Gyasi, Samantha Thomas, K. Houck, Whitney O. Lane, Rachel C. Blitzblau, et al. The effect of hospital volume on breast cancer mortality. Ann Surg 2018;267: $375-81$.

9. Cancer statistics. National Cancer Information Center, Ministry of Health and Welfare. http://www.cancer.go.kr. Accessed Aug 14th, 
2018.

10. Kim Z, Min SY, Yoon CS, Lee HJ, Lee JS, Youn HJ, et al. The basic facts of Korean breast cancer in 2011: results of a nationwide survey and breast cancer registry database. J Breast Cancer 2014;17:99-106.

11. Ko BS, Noh WC, Kang SS, Park BW, Kang EY, Paik NS, et al. Changing patterns in the clinical characteristics of Korean breast cancer from 1996-2010 using an online nationwide breast cancer database. J Breast Cancer 2012;15:393-400.

12. Kang SY, Kim YS, Kim Z, Kim HY, Lee SK, Jung KW, et al. Basic findings regarding breast cancer in Korea in 2015: data from a breast cancer registry. J Breast Cancer. 2018;21:1-10.

13. Autier P, Hery C, Haukka J, Boniol M, Byrnes G. Advanced breast cancer and breast cancer mortality in randomized controlled trials on mammography screening. J Clin Oncol 2009;27:5919-23.

14. Park EH, Min SY, Kim Z, Yoon CS, Jung KW, Nam SJ, et al. Basic facts of breast cancer in Korea in 2014. The 10-year overall survival progress. J Breast Cancer 2017;20:1-11.

15. Kim Z, Min SY, Yoon CS, Jung KW, Ko BS, Kang E, et al. The basic facts of Korean breast cancer in 2012: results from a nationwide sur- vey and breast cancer registry database. J Breast Cancer 2015;18: 103-11.

16. Mahmood U, Hanlon AL, Koshy M, Buras R, Chumsri S, Tkaczuk $\mathrm{KH}$, et al. Increasing national mastectomy rates for the treatment of early stage breast cancer. Ann Surg Oncol 2013;20:1436-43.

17. Yamashita H, Iwase H, Toyama T, Takahashi S, Sugiura H, Yoshimoto $\mathrm{N}$, et al. Estrogen receptor-positive breast cancer in Japanese women: trends in incidence, characteristics, and prognosis. Ann Oncol 2011;22:1318-25.

18. Devi CR, Tang TS, Corbex M. Incidence and risk factors for breast cancer subtypes in three distinct South-East Asian ethnic groups: Chinese, Malay and natives of Sarawak, Malaysia. Int J Cancer 2012; 131:2869-77.

19. Yip CH, Pathy NB, Uiterwaal CS, Taib NA, Tan GH, Mun KS, et al. Factors affecting estrogen receptor status in a multiracial Asian country: an analysis of 3557 cases. Breast 2011;20:S60-4.

20. Chen L, Linden HM, Anderson BO, Li CI. Trends in 5-year survival rates among breast cancer patients by hormone receptor status and stage. Breast Cancer Res Treat 2014;147:609-16. 